\title{
The characteristic polarized radio continuum distribution of cluster spiral galaxies
}

\author{
B. Vollmer ${ }^{1}$, M. Soida ${ }^{2}$, R. Beck ${ }^{3}$, M. Urbanik ${ }^{2}$, K. T. Chyży ${ }^{2}$, K. Otmianowska-Mazur ${ }^{2}$, \\ J. D. P. Kenney ${ }^{4}$, and J. H. van Gorkom ${ }^{5}$
}

${ }^{1}$ CDS, Observatoire astronomique de Strasbourg, 11 rue de l'université, 67000 Strasbourg, France e-mail: bvollmer@astro.u-strasbg.fr

2 Astronomical Observatory, Jagiellonian University, Kraków, Poland

3 Max-Planck-Insitut für Radioastronomie, Auf dem Hügel 69, 53121 Bonn, Germany

4 Yale University Astronomy Department, PO Box 208101, New Haven, CT 06520-8101, USA

5 Department of Astronomy, Columbia University, 538 West 120th Street, New York, NY 10027, USA

Received 20 December 2006 / Accepted 19 January 2007

ABSTRACT

\begin{abstract}
Deep observations are presented of the $6 \mathrm{~cm}$ polarized radio continuum emission of 8 Virgo spiral galaxies. All galaxies show strongly asymmetric distributions of polarized intensity with elongated ridges located in the outer galactic disk. These features are not found in existing observations of polarized radio continuum emission of field spiral galaxies, where the distribution of $6 \mathrm{~cm}$ polarized intensity is generally relatively symmetric and strongest in the interarm regions. We therefore conclude that most Virgo spiral galaxies and, most probably, the majority of cluster spiral galaxies show asymmetric distributions of polarized radio continuum emission due to their interaction with the cluster environment. The polarized continuum emission is sensitive to compression and shear motions in the plane of the sky and thus contains important information about the velocity distortions caused by these interactions.
\end{abstract}

Key words. galaxies: interactions - galaxies: ISM - galaxies: kinematics and dynamics - galaxies: magnetic fields radio continuum: galaxies

\section{Introduction}

Cluster spiral galaxies undergo different interactions that can change their gas distribution and content, stellar distribution, and morphology. These interactions can be tidal (with the cluster potential: see Byrd \& Valtonen 1990; Valluri 1999; or galaxygalaxy interactions: galaxy "harassment": Moore et al. 1998) or between the galaxy's ISM and the hot intracluster gas (ram pressure stripping: Gunn \& Gott 1972). To determine the nature of an interaction, multi wavelength observations are required. A tidal interaction leads to a distorted stellar and gas distribution observable in the optical (stars; gas: $\mathrm{H} \alpha$ ) and the radio (gas: $\mathrm{CO}$, HI). Spectroscopic observations of the ISM have the advantage of providing the distribution of the velocities along the line of sight. However, no direct information about the velocity components in the plane of the sky are accessible in this way. The distribution of polarized radio continuum emission can provide this information (Beck 2005).

Polarized radio continuum emission is due to relativistic electrons with density $n_{\mathrm{e}}$ gyrating around the regularly oriented, large-scale magnetic field $B: S_{\mathrm{PI}} \propto n_{\mathrm{e}} B^{2}$. The polarized radio continuum emission is enhanced in regions where shear and compression of regular or random magnetic fields occur. Polarized emission reveals compression regions much better than any other tracer, even outside starforming regions. From spectroscopic observations, non-circular motions of the order of $\sim 10 \mathrm{~km} \mathrm{~s}^{-1}$ induced by an interaction can be determined by a detailed analysis of a galaxy's velocity field (e.g. Schoenemakers et al. 1997). On the other hand, the distribution of polarized radio continuum emission represents a very sensitive tool for uncovering the transverse motions of the ISM (Beck 2005) even in the case of unfavorable inclinations (close to face-on). Therefore, the information contained in polarized radio continuum emission is complementary to that of $\mathrm{H} \alpha, \mathrm{CO}$, and $\mathrm{HI}$ observations. The total radio continuum emission is sensitive to the turbulent small-scale magnetic field, which is usually a factor of 2-5 larger than the regular large scale magnetic field in spiral arms and 1-2 times larger in the interarm regions at a typical resolution of a few 100 pc (Beck 2001). Whenever there is enhanced turbulence due to an enhanced star formation efficiency, the large scale magnetic field is diminished. The polarized radio continuum emission has to be observed at a frequency that is high enough to avoid significant Faraday rotation (typically $>2 \mathrm{GHz}$ ).

So far, only 2 Virgo spiral galaxies have been imaged with the VLA in polarized radio continuum emission, because this is very time consuming. An asymmetric pattern of magnetic spiral arms was found at 3 and $6 \mathrm{~cm}$ in NGC 4254 with the strongest polarization in the south, outside the optical arm (Chyży et al. 2006). In NGC 4522 Vollmer et al. (2004) also discovered an asymmetric distribution of the $6 \mathrm{~cm}$ polarized emission located at the eastern edge of the galactic disk, opposite the western extraplanar HI gas, which is pushed out of the galactic plane by ram pressure. Using the Effelsberg $100 \mathrm{~m}$ telescope, Soida et al. (1996) and Weżgowiec et al. (2007) found asymmetric distributions of polarized radio continuum emission at 3 and $6 \mathrm{~cm}$ in NGC 4254, NGC 4438, NGC 4501, NGC 4535, and NGC 4654. Encouraged by these results, we undertook a first high-resolution VLA survey of 8 Virgo spiral galaxies in polarized radio 
Table 1. Integration times and rms.

\begin{tabular}{llcccc}
\hline \hline Galaxy name & $m_{B}^{1}$ & $i^{2}$ & Dist. $^{3}$ & $\begin{array}{c}\text { Integration } \\
\text { time } \\
(\mathrm{h}: m i n)\end{array}$ & $\begin{array}{c}\mathrm{rms} \\
(\mu \mathrm{Jy} / \\
\text { beam })\end{array}$ \\
\hline NGC 4321 & 10.02 & $27^{a}$ & 4.0 & $7: 45$ & 9 \\
NGC 4388 & 11.87 & $77^{a}$ & 1.3 & $9: 25$ & 9 \\
NGC 4396 & 13.07 & $72^{b}$ & 3.5 & $8: 00$ & 9 \\
NGC 4402 & 12.64 & $74^{c}$ & 1.4 & $5: 00$ & 13 \\
NGC 4438 & 11.12 & $68^{a} / 85^{c}$ & 1.0 & $7: 45$ & 14 \\
NGC 4501 & 10.50 & $57^{a}$ & 2.0 & $3: 55$ & 11 \\
NGC 4535 & 10.73 & $43^{a}$ & 4.3 & $9: 00$ & 9 \\
NGC 4654 & 11.31 & $51^{a}$ & 3.4 & $7: 50$ & 9 \\
\hline
\end{tabular}

1 This research has made use of the GOLD Mine Database (Gavazzi et al. 2003); ${ }^{2}$ inclination angle; ${ }^{3}$ distance from M 87. ${ }^{a}$ From Cayatte et al. (1990); ${ }^{b}$ from NED; ${ }^{c}$ from Kenney et al. (1995).

continuum emission at 6 and $20 \mathrm{~cm}$. These galaxies span an order of magnitude in luminosity from 0.2 to $2 L^{*}$, have different inclinations, and are located at various distances between $1^{\circ}$ and $4^{\circ}$ from M 87. In this letter we report on the spectacular results of the observations of $6 \mathrm{~cm}$ polarized radio continuum emission and show that its distribution is different in cluster and field spiral galaxies.

\section{Observations}

The 8 Virgo spiral galaxies were observed between November 8 , 2005 and January 10, 2006 with the Very Large Array (VLA) of the National Radio Astronomy Observatory $(\mathrm{NRAO})^{1}$ in the $\mathrm{D}$ array configuration. The band passes were $2 \times 50 \mathrm{MHz}$. We used 3 C286 as the flux calibrator and $1254+116$ as the phase calibrator, the latter of which was observed every 40 min. Maps were made for both wavelengths using the AIPS task IMAGR with ROBUST $=3$. The final cleaned maps were convolved to a beam size of $18^{\prime \prime} \times 18^{\prime \prime}$. The bright radio source M 87 caused sidelobe effects enhancing the rms noise level of NGC 4438. We ended up with an rms level of the linear polarization, taken to be the mean rms in Stokes $Q$ and $U$, between 9 and $14 \mu \mathrm{Jy} / \mathrm{beam}$ (Table 1).

\section{Results}

The resulting maps of the $6 \mathrm{~cm}$ polarized radio continuum emission are shown in Fig. 1 as contours overlaid on optical $B$-band DSS images. The short lines delineate the orientation of the magnetic field uncorrected for Faraday rotation (hereafter referred to as " $B$-vectors"). Their length is proportional to the polarized intensity. In the $6 \mathrm{~cm}$ Effelsberg observations of NGC 4438, NGC 4501, NGC 4535, and NGC 4654, Weżgowiec et al. (2007) found a maximum Faraday rotation of $60 \mathrm{rad} / \mathrm{m}^{2}$. Therefore, the $B$-vectors represent the sky-projected regular field to an accuracy of $\pm 13^{\circ}$. We divided our sample into highly and mildly inclined spiral galaxies. Only the most distant galaxy from the cluster center, NGC 4321, shows the characteristic distribution of an unperturbed field spiral galaxy: the polarized radio continuum emission is mainly found in regions between the spiral arms, because turbulence linked to star formation in the spiral arms destroys the large scale magnetic field (see Sect. 1). There is, however, a north-south asymmetry that is also visible in the

${ }^{1}$ NRAO is a facility of National Science Foundation operated under cooperative agreement by Associated Universities, Inc. optical and HI images. All other Virgo spiral galaxies show a pronounced asymmetric distribution of the $6 \mathrm{~cm}$ polarized radio continuum emission with ridges in the outer part of the galactic disks:

In NGC 4501 we observe a strongly asymmetric distribution of polarized intensity with an extended maximum in the outer southwestern part of the disk, which coincides with a region of high column density HI (Cayatte et al. 1990). There is also a small polarized spot in the opposite part of the disk. $N G C 4535$ has an overall low emission of polarized radio continuum emission. The only region where we detect polarized emission is in the center and the southwest of the outer galactic disk located on the ring-like Hi distribution.

NGC 4654 shows an extended ridge of polarized emission in the southern part of the outer disk. We also find emission at the western and northern outer rim of the galactic disk. We did not detect any polarized emission in the southeastern extended HI tail (Phookun \& Mundy 1995).

$N G C 4396$ is a faint edge-on galaxy showing a ridge of polarized radio continuum emission in the northwestern part of the outer disk. Chung et al. (2007, in prep.) have detected an HI tail on this side of the galaxy extending to the northwest. In $N G C 4402$ we observe a strong maximum of polarized intensity in the western part of the disk where Crowl et al. (2005) detected extraplanar atomic hydrogen. There is no detection in the eastern outer disk. In addition, we observe extraplanar polarized emission above the disk plane in the northeast of the galactic disk, which coincides with the extended $20 \mathrm{~cm}$ radio continuum emission observed by Crowl et al. (2005). The observed HI and $20 \mathrm{~cm}$ continuum asymmetries are caused by ram pressure stripping.

$N G C 4388$ shows a ridge of polarized radio continuum emission in the southeastern part of the nearly edge-on disk. We observe a decreased polarized intensity to the southwest and northeast of the galaxy center. This is due to beam depolarization caused by the nuclear outflow that is visible in $\mathrm{H} \alpha$ emission (Veilleux et al. 1999). Thus, it is not excluded that the southeastern ridge of polarized intensity continues towards the west and then turns to the north.

NGC 4438 has strong tidal arms and shows the most surprising distribution of polarized radio continuum emission. Within the disk/bulge region, we observe a maximum to the southeast. However, most of the polarized emission is extraplanar and coincides with detections of extraplanar CO (Combes et al. 1988; Vollmer et al. 2005), HI (Cayatte et al. 1990), and $\mathrm{H} \alpha$ (Kenney et al. 1995).

\section{Discussion}

All 8 observed Virgo spiral galaxies show a strongly asymmetric distribution of polarized radio continuum emission with elongated ridges located in the outer galactic disk. Such polarized emission ridges are also observed in two other Virgo spiral galaxies: NGC 4254 (Chyży et al. 2006) and NGC 4522 (Vollmer et al. 2004). To investigate whether this kind of distribution is characteristic of Virgo cluster spiral galaxies, we will review the distribution of polarized radio continuum emission in field spiral galaxies. Since we have 4 edge-on galaxies in our sample, we first discuss our results for highly inclined field spiral galaxies.

Polarized radio continuum emission data at $20 \mathrm{~cm}$ of edge-on galaxies are available, e.g., for NGC 4631 (Hummel et al. 1988), NGC 891 (Hummel et al. 1991), NGC 4565 

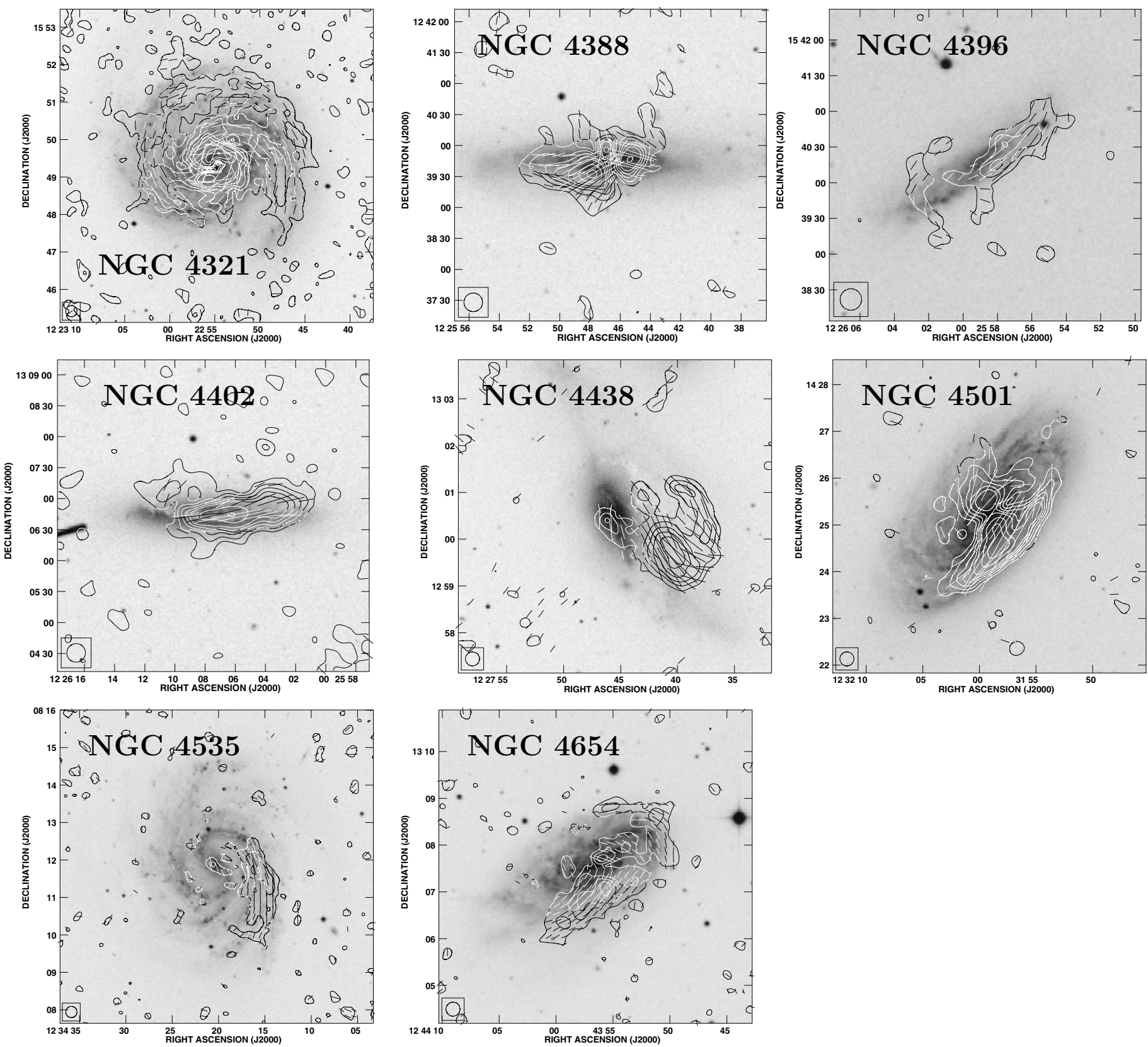

Fig. 1. $6 \mathrm{~cm}$ polarized intensities as contours and the vectors of the magnetic field uncorrected for Faraday rotation on DSS $B$ band images. The size of the lines is proportional to the intensity of the polarized emission. The contour levels are $(4,8,12,16,20,30,40,50,100,150) \times \eta \mu \mathrm{Jy}$ ( $~(\eta=8$ for NGC 4388, NGC 4396, NGC 4402, NGC 4654; $\eta=10$ for NGC 4321 and NGC 4535; $\eta=15$ for NGC 4438, NGC 4501). The beam $\left(18^{\prime \prime} \times 18^{\prime \prime}\right)$ is plotted in the lower left corner of the image.

(Sukumar \& Allen 1991), and NGC 5907 (Dumke et al. 2000). While NGC 4631, NGC 891, and NGC 4565 show fairly symmetric distributions of polarized emission, NGC 5907 has a polarized ridge in the southwest. However, one has to take into account that these data suffer from severe depolarization, because of the long wavelength and the long line of sight throughout the galactic disk. Thus one mainly sees the part of the galaxy that is closer to the observer. This effect already causes an asymmetry along the minor axis. Spiral arms can then cause asymmetries along the major axis; therefore, it is necessary to observe these galaxies at shorter wavelengths, where depolarization is small. Sukumar \& Allen (1991) and Tüllmann et al. (2000) observed the nearby edge-on galaxies NGC 891 and NGC 5775 at $6 \mathrm{~cm}$ with the VLA and found fairly symmetric distributions of polarized emission. Dumke et al. (2000) observed NGC 5907 at $6 \mathrm{~cm}$ in polarization. Despite the asymmetric distribution of the polarized emission at $20 \mathrm{~cm}$, they found that the $6 \mathrm{~cm}$ polarized emission distribution is almost symmetric. Thus, we conclude that highly inclined field galaxies can have heavily asymmetric polarized emission distributions caused by depolarization at $20 \mathrm{~cm}$, but not at $6 \mathrm{~cm}$.

A compilation of the imaging polarization measurements of 17 nearby galaxies at $6 \mathrm{~cm}$ with the VLA, ATCA, and the Effelsberg $100 \mathrm{~m}$ telescope is available on the website of 
the Max-Planck-Institut für Radioastronomie Bonn ${ }^{2}$. At $6 \mathrm{~cm}$ wavelength, none of the non-interacting, isolated galaxies (NGC 2276 has a companion, M 101 is a member of a group) shows prominent maxima or ridges in the polarized radio continuum emission on the outer edge of the galactic disk as it is observed in the extended sample of 10 Virgo spiral galaxies. We conclude that these maxima are characteristic of cluster and probably group spiral galaxies.

Since all asymmetric emission ridges are located in the outer galactic disks, they are most likely due to external influences of the cluster environment on the galaxies. These influences can be of a tidal or hydrodynamic nature (see Sect. 1). Thus the distribution of polarized radio continuum emission contains important information about the velocity distortions caused by the interaction of a spiral galaxy with its cluster environment. In addition, this information is complementary to that of $\mathrm{CO}$ or HI data cubes, because shear or compression motions can have dominant velocity components in the plane of the sky. It is very difficult, if not impossible, to predict the distribution of polarized radio continuum emission on the basis of $\mathrm{CO}$ or $\mathrm{HI}$ observations, because of the complex evolution of the magnetic field (induction equation) and beam depolarization effects. It is therefore necessary to do detailed MHD modelling of individual galaxies for direct comparison with observations. In OtmianowskaMazur \& Vollmer (2003), we demonstrated the feasibility of this method and we applied it successfully in Vollmer et al. (2004) and Soida et al. (2006) to the Virgo spiral galaxies NGC 4522 and NGC 4654, respectively. In both cases the comparison between the observed and the modelled polarized radio continuum emission distribution confirm the scenarios based on detailed numerical simulations and deep HI observations.

Based on a sample of 10 Virgo spiral galaxies observed with the VLA, we conclude that the $6 \mathrm{~cm}$ polarized radio continuum emission distribution in Virgo spiral galaxies is different from that of field spiral galaxies (Beck 2005), i.e. the distribution is strongly asymmetric with elongated ridges in the outer part of the galactic disks. These data contain important information on the influence of the cluster environment on a spiral galaxy. Quantitative information can be extracted by a detailed comparison with MHD models that include tidal and hydrodynamic (ram pressure) interactions.

Acknowledgements. This work was supported by the Polish-French (ASTROLEA-PF) cooperation program and by the Polish Ministry of Sciences and Higher Education grant PB 378/P03/28/2005 and 2693/H03/2006/31.

\section{References}

Beck, R. 2001, SSRv, 99, 243

Beck, R. 2005, Cosmic Magnetic Fields, ed. R. Wielebinski, \& R. Beck (Berlin, Springer). Lecture notes in Physics, 664, 41

Byrd, G., \& Valtonen, M. 1990, ApJ, 350, 89

Cayatte, V., van Gorkom, J. H., Balkowski, C., \& Kotanyi, C. 1990, AJ, 100, 604 Chyży, K. T., Ehle, M., \& Beck, R. 2006, AN, 327, 501

Combes, F., Dupraz, C., Casoli, F., \& Pagani, L. 1988, A\&A, 203, L9

Crowl, H. H., Kenney, J. D. P., van Gorkom, J. H., \& Vollmer, B. 2005, AJ, 130, 65

Dumke, M., Krause, M., \& Wielebinski, R. 2000, A\&A, 355, 512

Gavazzi, G., Boselli, A., Donati, A., Franzetti, P., \& Scodeggio, M. 2003, A\&A, 400,451

Hummel, E., Lesch, H., Wielebinski, R., \& Schlickeiser, R. 1988, A\&A, 197, L29

Hummel, E., Beck, R., \& Dahlem, M. 1991, A\&A, 248, 23

Kenney, J. D. P., Rubin, V. C., Planesas, P., \& Young, J. S. 1995, ApJ, 438, 135

Moore, B., Lake, G., \& Katz, N. 1998, ApJ, 495, 139

Otmianowska-Mazur, K., \& Vollmer, B. 2003, A\&A, 402, 879

Phookun, B., \& Mundy, L. G. 1995, ApJ, 453, 154

Schoenemakers, R. H. M., Franx, M., \& de Zeeuw, P. T. 1997, MNRAS, 292, 349

Soida, M., Urbanik, M., \& Beck, R. 1996, A\&A, 312, 409

Soida, M., Otmianowska-Mazur, K., Chyzy, K., \& Vollmer, B. 2006, A\&A, 458, 727

Sukumar, S., \& Allen, R. J. 1991, ApJ, 382, 100

Tüllmann, R., Dettmar, R.-J., Soida, M., Urbanik, M., \& Rosa, J. 2000, A\&A, 364, L36

Valluri, M. 1993, ApJ, 408, 57

Veilleux, S., Bland-Hawthorn, J., Cecil, G., Tully, R. B., \& Miller, S. T. 1999, ApJ, 520, 111

Vollmer, B., Beck, R., Kenney, J. P. D., \& van Gorkom, J. H. 2004, AJ, 127, 3375

Vollmer, B., Braine, J., Combes, F., \& Sofue, Y. 2005, A\&A, 441, 473

Weżgowiec, M., Urbanik, M., Vollmer, B., et al. 2007, A\&A submitted

\footnotetext{
${ }^{2}$ http://www.mpifr-bonn.mpg.de/staff/ wsherwood/mag-fields.html
} 